Sharif University of Technology
Scientia Iranica
SCIENTIA
I RAN I CA

\title{
Seismic performance evaluation of jacket-type offshore platforms using endurance time method considering soil-pile-superstructure interaction
}

\author{
H. Hasani*, A.A. Golafshani and H.E. Estekanchi \\ Department of Civil and Environmental Engineering, Sharif University of Technology (SUT), Tehran, P.O. Box 11155-1639, Iran.
}

Received 10 November 2015; accepted 2 August 2016

KEYWORDS
Endurance time
method;
Soil-pile-
superstructure
interaction;
Single pile;
Jacket offshore
platform;
Seismic assessment.

\section{KEYWORDS}

method;

Soil-pile-

superstructure

interaction;

Seismic assessment.

\begin{abstract}
In this paper, application of Endurance Time (ET) method in nonlinear seismic analysis of offshore pile supported systems has been studied. The ET method is a time-history analysis in which structures are subjected to intensifying artificial acceleration functions. The ET method reduces complexity and computational demand of conventional nonlinear seismic analysis, and it provides response at different seismic levels in a single ET analysis. The aforementioned methodology has been applied to a typical model of single pile and then to a functional jacket offshore platform in Persian Gulf region. Seismic response of aforesaid models by ET method has been compared with conventional time-history method. The results indicate that ET method is reliable in capturing seismic response of offshore platforms supported on piles with an acceptable accuracy.
\end{abstract}

(C) 2017 Sharif University of Technology. All rights reserved.

\section{Introduction}

The main objective of seismic design is to provide safe structures at reasonable cost. Offshore platforms have been used in petroleum activity for decades, and these types of structures are considered as highly important in oil industry. Special efforts are required to operate them continuously over time for economic and environmental reasons. Therefore, it is necessary to pay special attention to the analysis of these types of structures. Current methods for seismic analysis of these structures have a number of problems such as being complex and time consuming. In this paper, the ET method on the offshore structures with deep foundation (pile), such as offshore platforms, has been investigated.

\footnotetext{
* Corresponding author. Fax: +982122415800 E-mail addresses: h_hasani@alum.sharif.edu (H. Hasani), golafshani@sharif.edu (A.A. Golafshani); stkanchi@sharif.edu (H.E. Estekanchi)
}

The most common offshore platforms, which are used in the oil industry, are Jacket-Type Offshore Platform (JTOP) which consists of the jacket structure, pile foundation, and its supporting soil. During earthquakes, these components act with each other to produce the global response of the JTOP so that the shear waves propagate through different soil layers and lead to kinematic forces to the pile foundation; subsequently, the pile shaking induces inertial force in the jacket structure. Hence, the seismic response of structures with pile is strongly related to non-linear behavior of piles. The ET method, however, was investigated on simplified models of JTOPs regardless of their piles so far [1].

Estekanchi et al. introduced the ET method [2] as a new seismic analysis method. The ET method has been applied in the linear and nonlinear seismic analyses of several structures which are built on soil such as steel frames [3,4], concrete gravity dams [5], unanchored steel storage tanks [6], steel liquid storage tanks [7], and shell structures [8]. However, the 
reliability and level of accuracy of the method, in the case of pile supported structures, have not been examined. It is important to extend these studies to the area of pile supported structures such as jacket-type offshore structures, since the presence of different soil layers results in complex models with a special type of ground motion filtering effects that is not common in other types of structures.

In this study, the ability of ET method in estimating seismic behavior of offshore structures supported with piles is investigated. First, the concept of ET method is explained. In addition to ET analysis, timehistory analysis is conducted for comparative analysis of the results obtained from the two methods. For this purpose, the authors selected two case studies: first, a Single Pile (SP) was tested by the centrifuge at the University of California at Davis under an earthquake loading [9]; second, a JTOP in the Persian Gulf. Afterwards, these structures with soil-pile-superstructure interaction have been modeled in Open System for Earthquake Engineering Simulation (OpenSees) [10]; moreover, for non-linear one-dimensional site response analysis, DEEPSOIL [11] has been used.

\section{Endurance time concept}

The ET method is a dynamic analysis procedure which uses artificial intensifying acceleration as the loading functions. By using this method, the entire range of excitation levels of interest can be covered in a single numerical or experimental simulation, thus significantly reducing the computational demand as compared to full nonlinear response-history analyses. ET analysis makes it easy to compare the improvements or disadvantages resulting from changes in the design parameters. Due to the dynamic nature of this method, structural modeling and loading on structure can be accomplished with an acceptable precision.

In the ET procedure, structures are subjected to a set of artificial intensifying acceleration functions; for example, an ET acceleration function (ETA20en01) is shown in Figure 1. These ET accelerograms are designed in a manner which has a linear relationship with intensity of acceleration and displacement response spectrum as shown in Eqs. (1) and (2):

$$
\begin{aligned}
& S_{a T}(T, t)=\frac{t}{t_{\text {Target }}} S_{a c}(T), \\
& S_{u T}(T, t)=\frac{t}{t_{\text {Target }}} S_{a c}(T) \times \frac{T^{2}}{4 \pi^{2}},
\end{aligned}
$$

where $S_{a T}(T, t)$ represents target acceleration response at time $t, T$ stands for the period of free vibration, $S_{a c}(T)$ is designated as the template design acceleration spectrum, and $S_{u T}(T, t)$ is the target displacement response at time $t$. To obtain an ET Acceleration

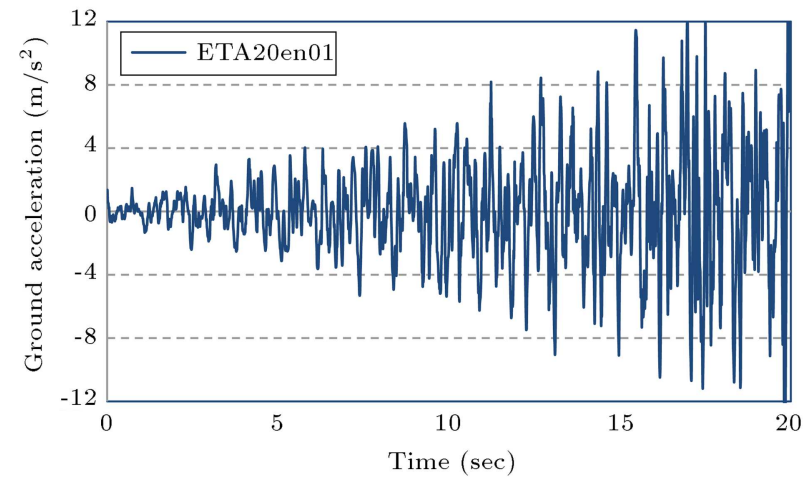

Figure 1. The ETAen01 accelerogram.

Function (ETAF) by these features, it is formulated as an unconstrained optimization problem in the time domain as follows:

$$
\text { Minimize } \begin{aligned}
F\left(a_{g}\right)= & \int_{0 t}^{T_{\max }} \int_{0}^{t_{\max }}\left\{\left[S_{a}(T, t)\right.\right. \\
& \left.-S_{a T}(T, t)\right]^{2}+\alpha\left[S_{u}(T, t)\right. \\
& \left.\left.-S_{u T}(T, t)\right]^{2}\right\} d t d T,
\end{aligned}
$$

where $F\left(a_{g}\right)$ represents the ETAF being sought, and $\alpha$ stands for an optimization weighting parameter set to 1.0 in this study.

Preliminary concepts of this method can be introduced by a hypothetical shaking table test in which there are three offshore platforms, and intensifying acceleration function of ET is applied to them. In the beginning, all three platforms are stable up to the 5th second; while intensity of excitation gradually increases, amplitude of structures becomes large as well due to the increasing demand for acceleration function. Eventually, platforms show non-linear behavior after elastic deformations. As shown in Figure 2, platform number 1 is stronger than platform number 3 , and number 3 is stronger than number 2. If the ETAF is scaled such that response spectrum in the 10th second is in accordance with ELE [12] event and the 15th second is in accordance with ALE [12] event, it can be concluded that platform number 3 is a suitable one; platform number 2 is weak, and platform number 1 is non-economic.

\section{Model description}

\subsection{Single pile}

All experimental results related to SP model have been extracted from CSP4 experimental centrifuge test done by Wilson et al. (1997) [13]. All results in study of Wilson et al. (1997) [13] were in prototype units. SP was in a Flexible Shear Beam [14], and soil profile was composed of two horizontal layers. The lower layer was 


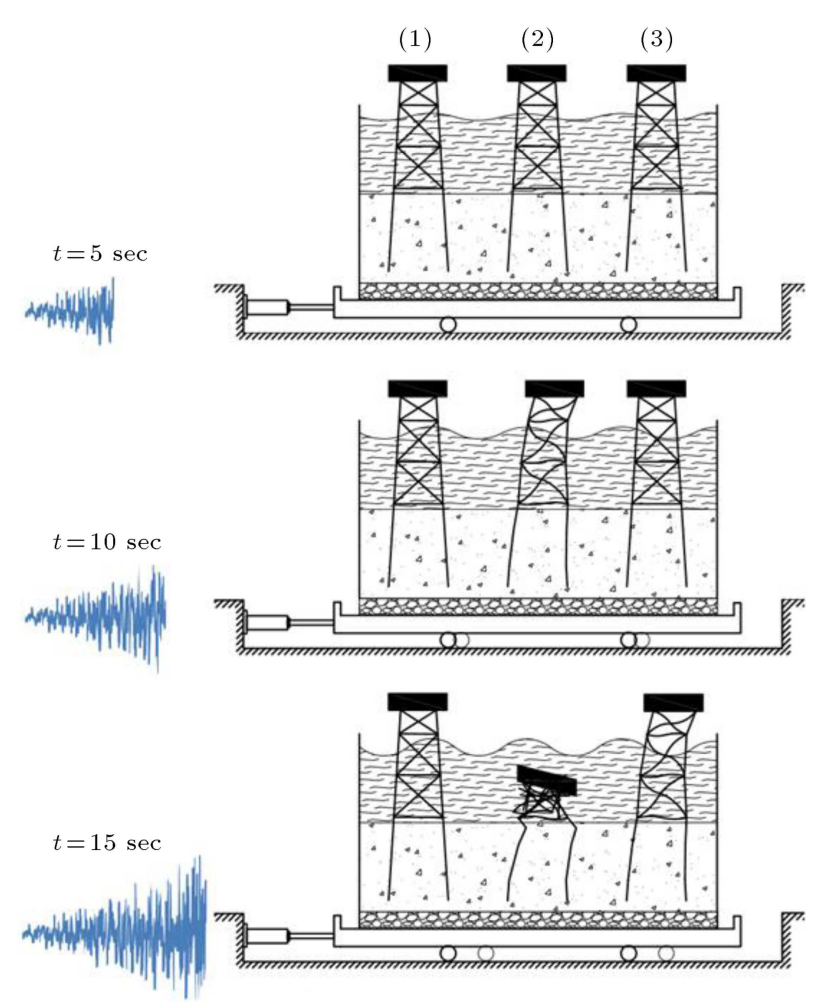

Figure 2. Hypothetical test of shaking table.

fine uniformly graded Nevada sand with a $C_{u}$ of 1.5 , $D_{50}$ of 15 , dry density of $66 \mathrm{Mg} / \mathrm{m}^{3}$, and $D_{r} 75-80 \%$. The upper layer was very soft clay from Bay mud with LL of $88 \%$, PI of $48 \%$, and a moist soil (water content= $140 \%)$; moreover, this layer was placed in four equal layers; in addition, each layer was separated by a sheet of filter to accelerate consolidation. $c_{u}$, according to Boulanger et al. (1999) [9], was considered to be equal to $c_{u}=0.45 \sigma^{\prime}{ }_{v c}$. Also, the chosen SP consisted of a superstructure with mass of $49.1 \mathrm{Mg}$ attached to the top of SP at elevation of $3.81 \mathrm{~m}$. This aluminum SP in the test was approximately equivalent to a steel pile with diameter of $0.67 \mathrm{~m}$, thickness of $19 \mathrm{~mm}$, mass per unit length of $0.37 \mathrm{Mg} / \mathrm{m}$, and EI equal to $417{\mathrm{MN} . \mathrm{m}^{2}}^{2}$ (Figure 3).

In CSP4 experiment, Kobe earthquake (1995) for Port Island Station, whose noises were removed and scaled to $0.055 \mathrm{~g}$, was entered to centrifuge table.

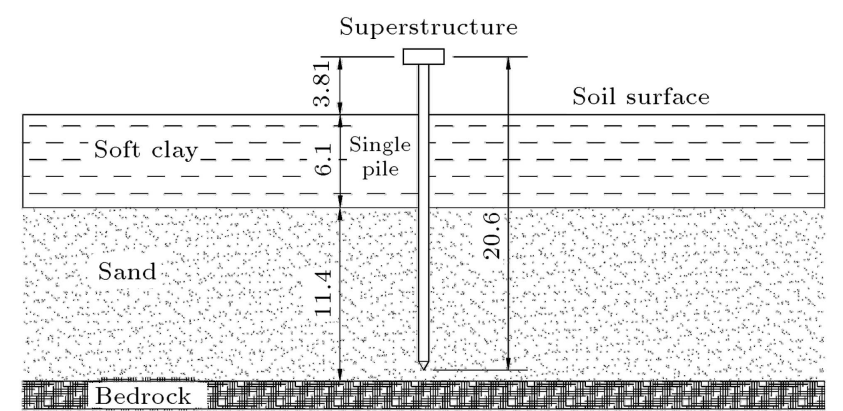

Figure 3. SP supported structure in CSP4 test.

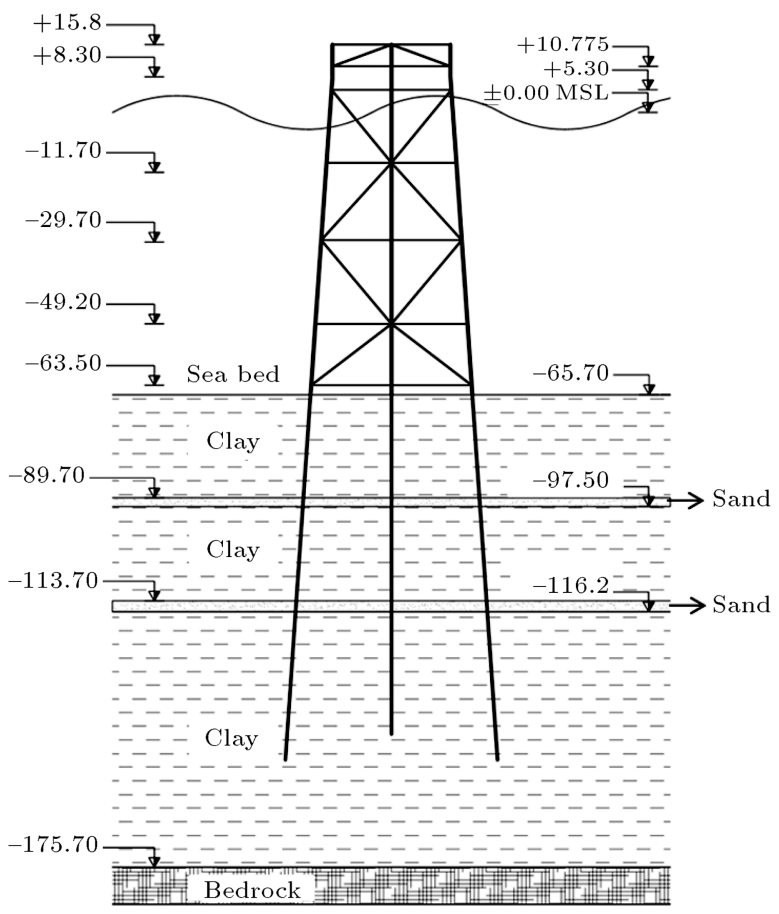

Figure 4. General view of the SPD2 frame.

\subsection{Functional jacket type offshore platform}

In this study, SPD2 as a JTOP located in South Pars Gas field Phase-1 in Persian Gulf region was considered herein. This platform is a six-legged jacket, and there is a grouted pile at the end of each leg. The four piles at the corners of longitudinal faces of platform are extended $85 \mathrm{~m}$, and two intermediate piles are extended $79 \mathrm{~m}$ below the mud line elevation. The jacket dimensions in the horizontal plane at the top and bottom (mud line) are about $16.00 \mathrm{~m} \times 27.50 \mathrm{~m}$ and $23.4 \mathrm{~m} \times 37.7 \mathrm{~m}$. The mean water depth is $65 \mathrm{~m}$ (Figure 4).

Furthermore, bedrock elevation in this area is nearly at $110 \mathrm{~m}$ depth beneath the mud line, and profile of median shear wave velocity in the vicinity of this jacket is shown in Figure 5.

\section{Numerical simulations}

\subsection{Pile-soil interaction}

Regarding these models, BNWF was employed to consider nonlinear pile-soil interaction. In the BNWF model, movements around pile, where pile-soil interaction occurs, is called near-field movement, and movements far from the pile are called far-field or free-field movement. We simulated far-field movements by $1-D$ wave propagation analysis program for geotechnical response analysis of deep soil deposits (DEEPSOIL) [11]; moreover, for near-field movements, we simulated these movements by independent springs ( $\mathrm{p}-\mathrm{y}, \mathrm{t}-\mathrm{z}$, and $\mathrm{q}-\mathrm{z}$ ) connected horizontally and vertically to pile elements. $\mathrm{p}-\mathrm{y}$ springs indicate soil reaction 


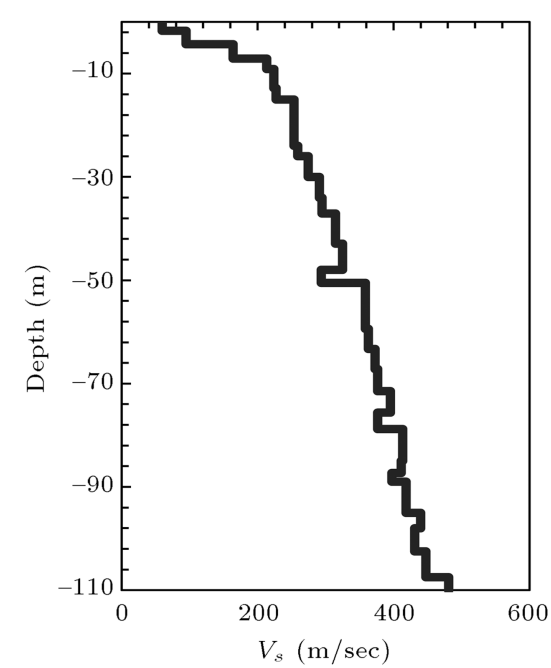

Figure 5. Profile of median shear wave velocity for site of South Pars Gas field in the vicinity of SPD2 location used in site response analyses [28].

force versus lateral displacement response of pile in different layers; $t-z$ springs consider the shear force transferred between the soil and the pile in various depths, and q-z springs provide end bearing resistance. The concept of dynamic BNWF model is shown in Figure 6.

In addition, the dynamic model used for p$\mathrm{y}$ elements in this paper is illustrated in Figure 7. Displacement time history calculated by DEEPSOIL was applied to p-y element along the pile. The BNWF model can take the variation of soil properties with depth, nonlinear soil behavior, dissipating energy by hysteric damping for radiation damping [15], and

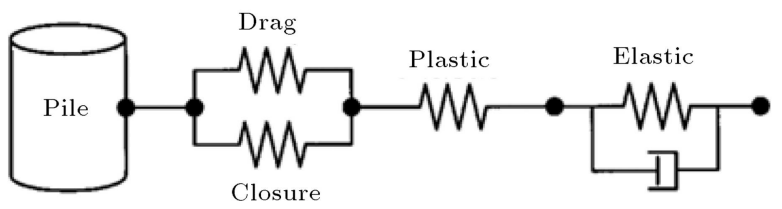

Figure 7. Components of nonlinear p-y element [9].

gapping effect [16] into account. Penzien et al. [17], Kagawa and Kraft [18], Nogami et al. [19], Boulanger et al. [9], and Naggar and Bentley [20] proposed several models of the BNWF. In this study, nonlinear p-y material with gapping capability (Figure 7), in addition to t-z and $\mathrm{q}-\mathrm{z}$ materials, was modeled using the element described by Boulanger et al. (1999) [9] The characteristics of these springs were estimated by employing recommendations in API RP-2A WSD [21].

\subsection{Free-field site response analysis}

\subsubsection{Single pile}

According to the recommendations of Boulanger et al. [9], small-strain shear modulus $G_{\max }$ of sand was calculated by Eq. (4), which was obtained by Seed and Idris [22], and $G_{\max }$ of clay was considered to be equal to $G_{\max / c_{u}}=380$ :

$$
\frac{G_{\max }}{P_{\text {atm }}}=21.8 K_{2, \max } \sqrt{\frac{\sigma_{m}^{\prime}}{P_{\text {atm }}}},
$$

where $K_{2, \max }=65, \sigma_{m}^{\prime}=\left(1+2 K_{0}\right) \sigma_{v c}^{\prime} / 3$, and $K_{0}=$ 0.6 .

To obtain a precise match between numerical nonlinear model of deep soil deposit and experimental results, MRDF pressure-dependent hyperbolic model was used to find the fitting parameters [23]. Further-

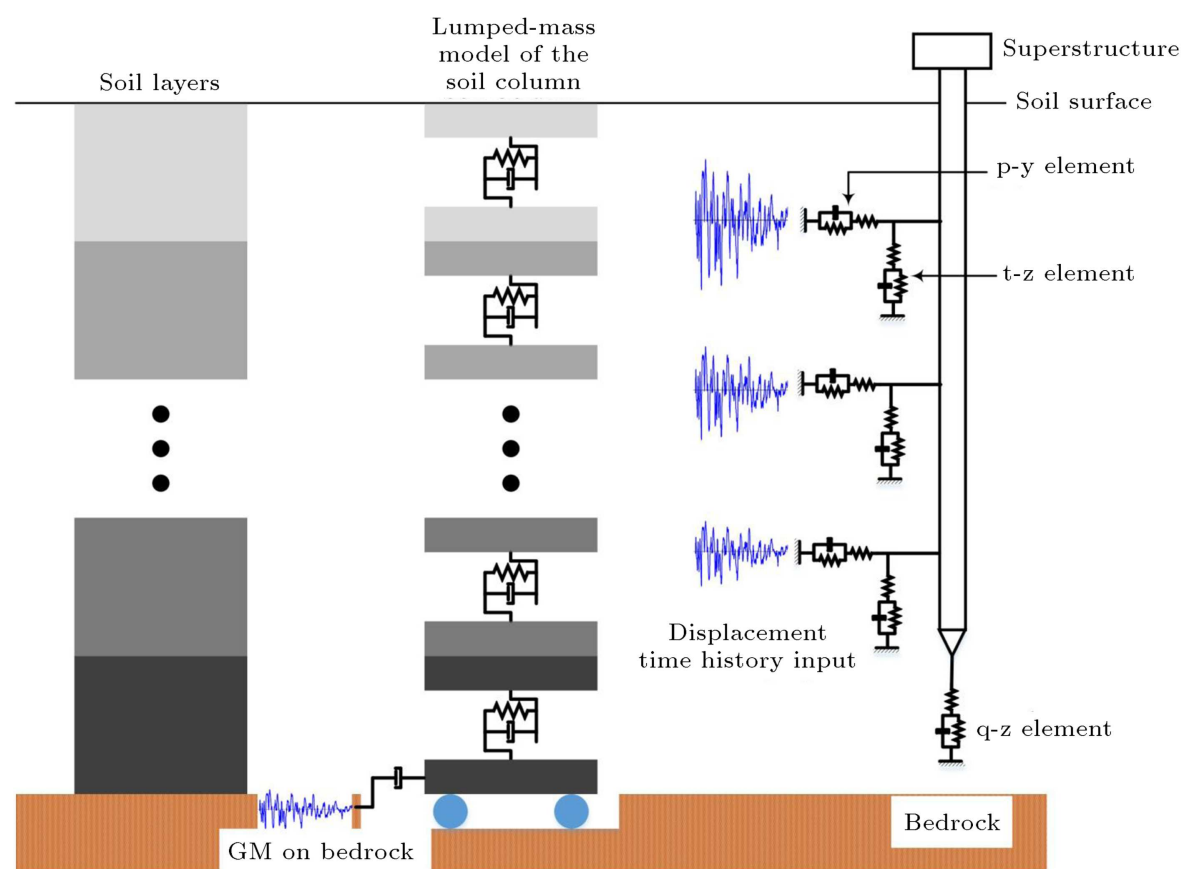

Figure 6. A schematic concept of soil-pile-structure interaction dynamic BNWF model used in this research. 
more, for curve fitting procedure, the MRDF-Darendeli was used. Considering the clay, Darendeli [24] target curves, and for the sand, Vucetic \& Dobri [25] target curves for damping versus strain and $G / G_{\max }$ versus strain were chosen.

Forty-nine layers of soil were considered for this model since the maximum frequency which can be propagated from thick layers of soil is small; moreover, using fewer numbers of layers (thicker layers) filters high frequency waves in low spectral accelerations [26].

Besides, SP was in Flexible Shear Beam; hence, the total unit weight of the soil was increased for considering the lateral forces of the rings according to the suggestion of Van Laak et al. [27].

\subsubsection{Functional offshore platform}

As demonstrated in Figure 5, median shear wave velocity for South Pars Gas field site in the vicinity of SPD2 location was used, which was calculated by Tabandeh [28]. Afterwards, the authors used pressuredependent hyperbolic model for soil column behavior of this model, and Darendeli's models [24] were selected to be the reference curves.

\subsection{Structure modeling}

Both SP and SPD2 were modeled in 2D; additionally, fiber section (in combination with beam-column) was used for modeling.

For the JTOP, the initial errors of constructional offshore platform were accounted with value of $0.002 \mathrm{~L}$. Moreover, all topside loads were applied to the main joints as equivalent point loads, and in this model, the added mass effect was considered for hydrodynamic effects [21].
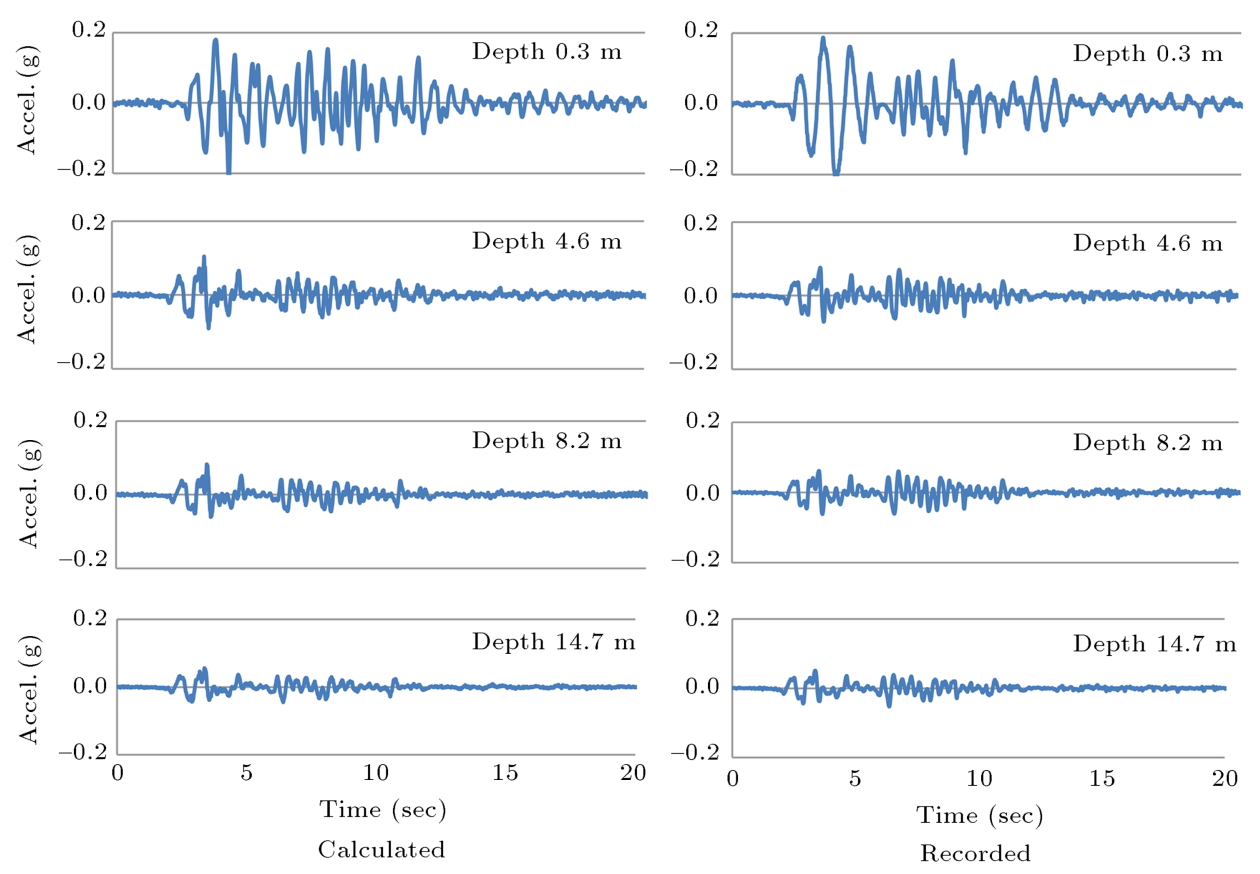

Figure 8. Recorded and calculated accelerations in soil profile during event B (Kobe motion) in CSP4.

\subsection{Comparison of numerical and experimental results for a single pile}

The results of experimental centrifuge tests which were done by Wilson (1997) [13], and nonlinear dynamic analyses performed for verification on numerical model of SP are shown in Figures 8 to 10 . All results of centrifuge tests were extracted from University of California at Davis website [29]. As can be seen in Figures 8 and 9, there is acceptable agreement between recorded and calculated accelerations in both time history acceleration and $S_{a}$ in different soil layers, and this satisfactory agreement is observed in some parts of superstructure as well (Figure 10).

\section{Comparison of acceleration response spectrums in ET analysis with those in conventional time-history analysis}

Spectral response accelerations $\left(S_{a}\right)$ of these models were calculated according to the simplified seismic action procedure of ISO 19901-2 [12]. SPD2 has exposure level of L2 and $C_{r}$ of 1.4 [30], and because this JTOP is located in the South Pars region of Persian Gulf, it is in the site seismic zone of 3 . It was assumed that SP belonged to a structure as important as SPD2 and was located in the same area; hence, SP had the same exposure level, $C_{r}$, and seismic zone. Therefore, for both SP and SPD2, ALE and ELE $S_{a}$ related to ISO 19901-2 were identical (as shown in Figures 11 to 14).

Seven horizontal Ground Motion (GM) records were selected from PEER Ground Motion Databasea [31]; Table 1 shows the specifications of these GMs. 

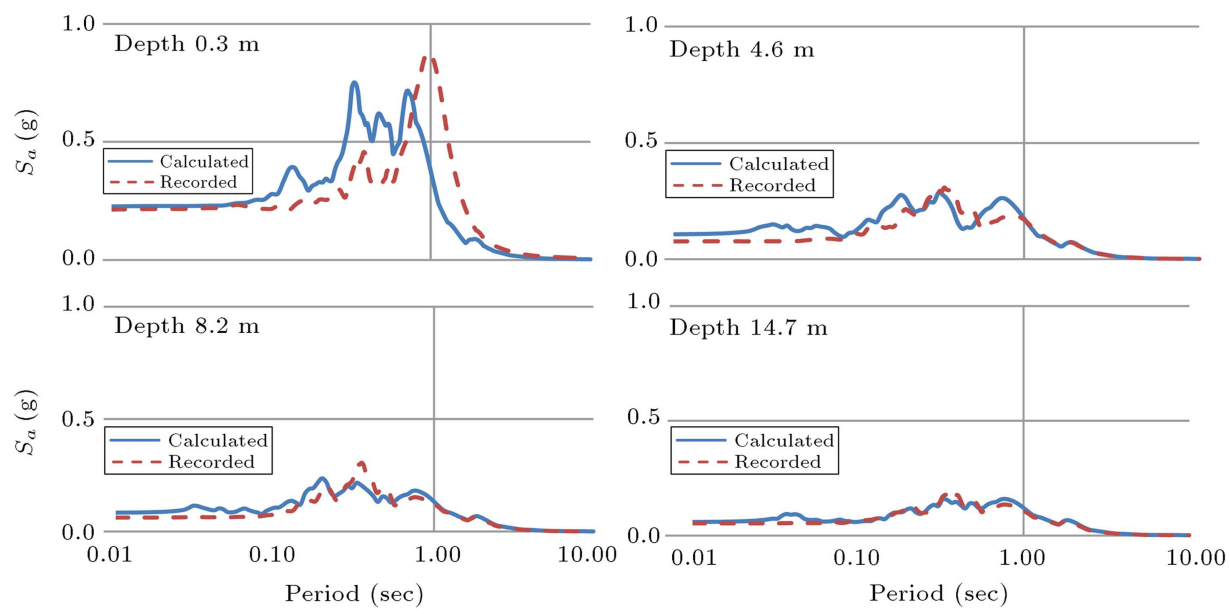

Figure 9. ARS (5\% damping) in soil profile.
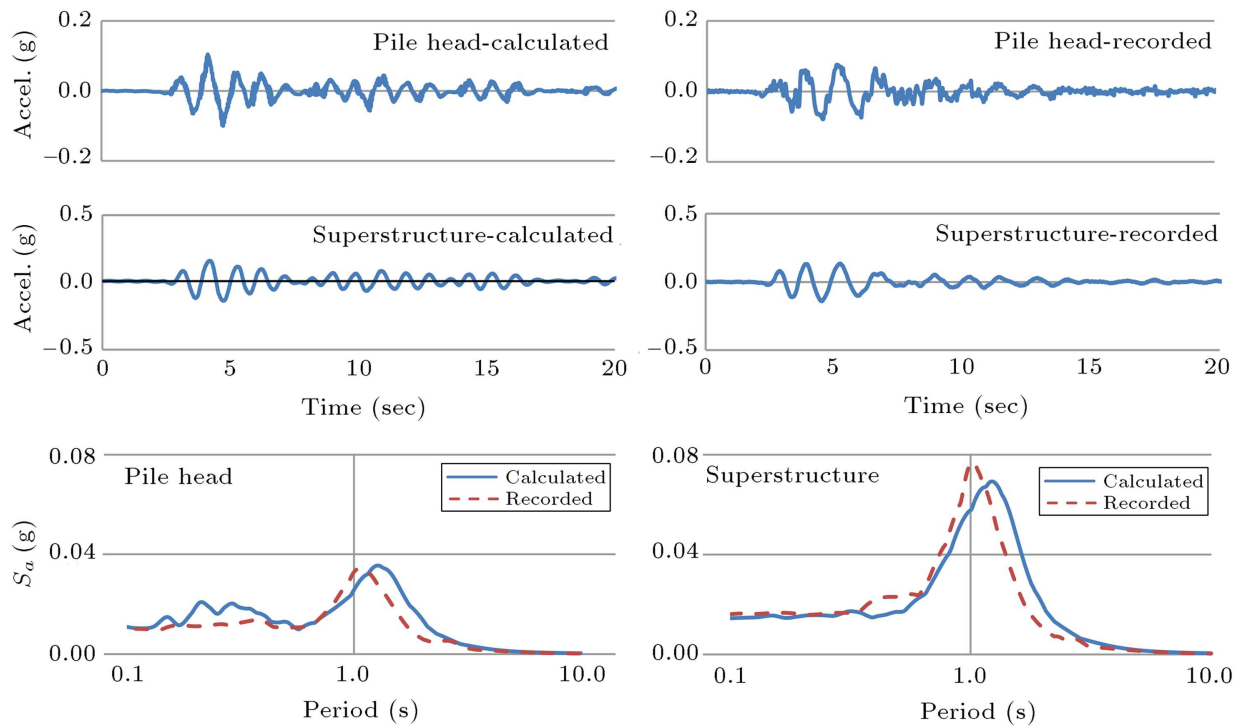

Figure 10. Accelerations and ARS (5\% damping) for pile head and superstructure of structure SP during event B (Kobe motion) in CSP4 test.

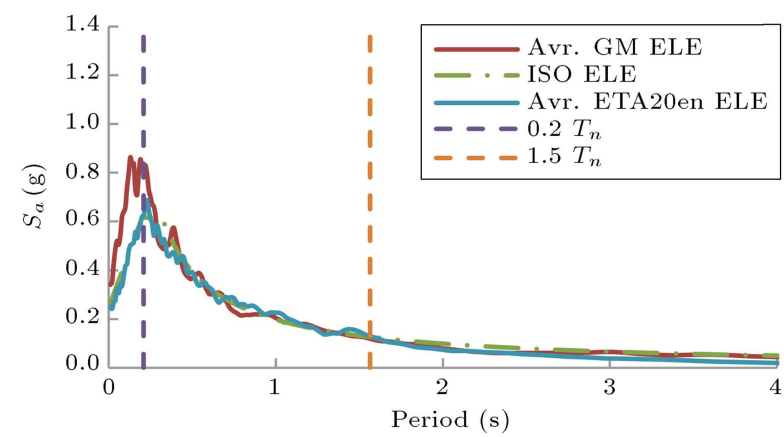

Figure 11. ELE ARS of ISO 19901 code, average GM, and ETAF for SP.

For scaling of these records, the method of ISO 19901-2 Code [12] was used. According to this method, standard GM is scaled so that scaled GM has the same $S_{a}$ with $S_{a}$ related to ISO in fundamental period (for SP $1.04 \mathrm{sec}$ and for SPD2 $1.93 \mathrm{sec}$ ); the scaling factors

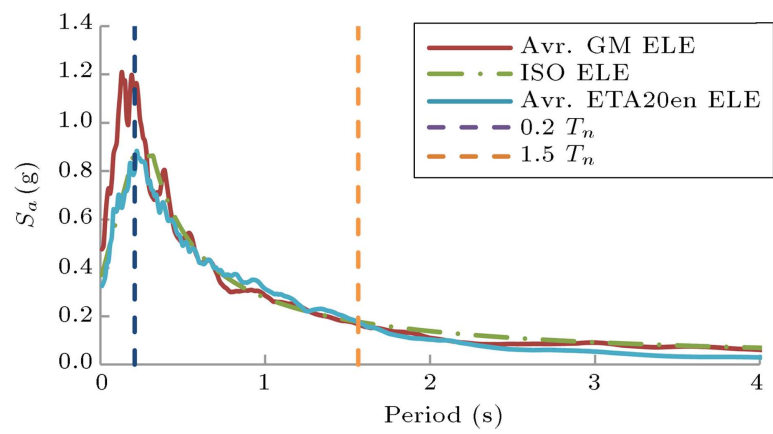

Figure 12. ALE ARS of ISO 19901 code, average GM, and ETAF for SP.

which were obtained by this method can be seen in Table 2 .

According to the ET methodology, the target time in the ETAF should be found so that the acceleration spectra of each ETAF until target time 
Table 1. Specifications of GMs.

\begin{tabular}{cccccc}
\hline NGA\# & Event & Year & Station & Magnitude & VS30 (m/s) \\
\hline $\mathbf{7 8 8}$ & Loma Prieta & 1989 & Piedmont Jr High & 6.93 & 895.4 \\
$\mathbf{7 8 9}$ & Loma Prieta & 1989 & Point Bonita & 6.93 & 1315.9 \\
$\mathbf{8 9 1}$ & Landers & 1992 & Silent Valley-Poppet Flat & 7.28 & 684.9 \\
$\mathbf{8 9 7}$ & Landers & 1992 & Twenty nine Palms & 7.28 & 684.9 \\
$\mathbf{9 4 6}$ & Northridge-01 & 1994 & Antelope Buttes & 6.69 & 821.7 \\
$\mathbf{1 0 4 1}$ & Northridge-01 & 1994 & Mt Wilson-CIT Seis Sta & 6.69 & 821.7 \\
$\mathbf{1 0 9 6}$ & Northridge-01 & 1994 & Wrightwood-Jackson Flat & 6.69 & 821.7 \\
\hline
\end{tabular}

Table 2. Scale factors of GMs for SP and SPD2.

\begin{tabular}{cccccc}
\hline & \multicolumn{2}{c}{ SP } & & \multicolumn{2}{c}{ SPD2 } \\
\cline { 2 - 3 } \cline { 6 - 7 } NGA\# & $\begin{array}{c}\text { ELE } \\
\text { scale } \\
\text { factor }\end{array}$ & $\begin{array}{c}\text { ALE } \\
\text { scale } \\
\text { factor }\end{array}$ & & $\begin{array}{c}\text { ELE } \\
\text { scale } \\
\text { factor }\end{array}$ & $\begin{array}{c}\text { ALE } \\
\text { scale } \\
\text { factor }\end{array}$ \\
\hline $\mathbf{7 8 8}$ & 1.99 & 2.78 & & 2.028 & 2.839 \\
$\mathbf{7 8 9}$ & 0.84 & 1.18 & & 0.850 & 1.190 \\
$\mathbf{8 9 1}$ & 8.45 & 11.83 & & 8.310 & 11.634 \\
$\mathbf{8 9 7}$ & 8.45 & 11.84 & & 8.244 & 11.542 \\
$\mathbf{9 4 6}$ & 3.90 & 5.46 & & 3.880 & 5.432 \\
$\mathbf{1 0 4 1}$ & 4.89 & 6.85 & & 4.882 & 6.833 \\
$\mathbf{1 0 9 6}$ & 2.60 & 3.65 & & 2.484 & 3.478 \\
\hline
\end{tabular}

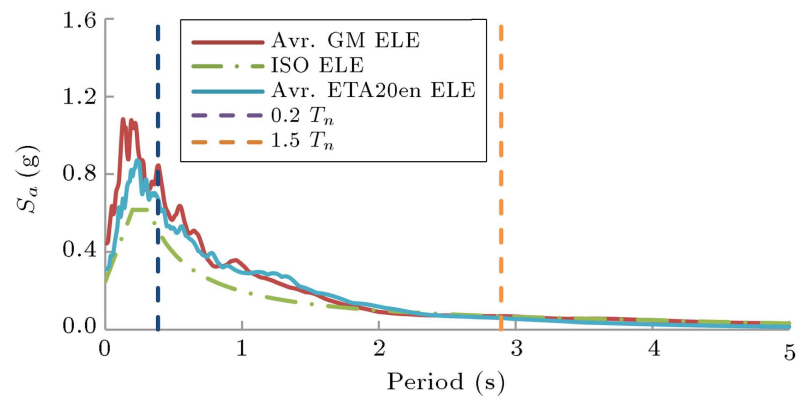

Figure 13. ELE ARS of ISO 19901 code, average GM, and ETAF for SPD2.

become compatible with the average spectrum of GMs between $0.2 T_{n}$ and $1.5 T_{n}$. To calculate the time, we considered the linear relation between acceleration response spectrum and recorded length in the ET method. In the initial calculation, the target

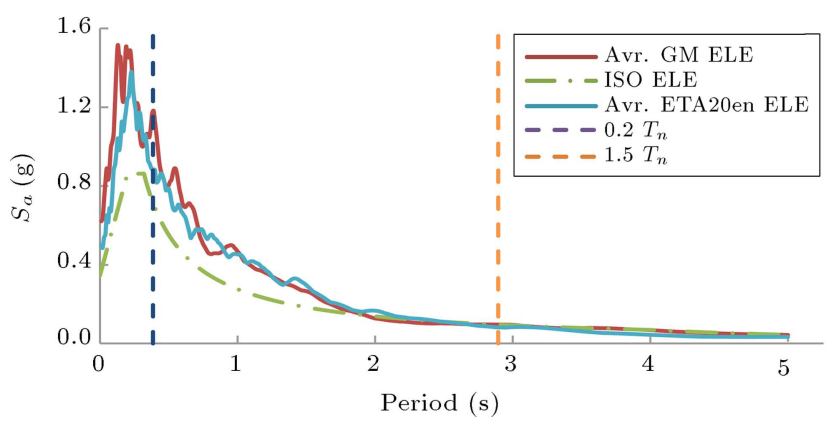

Figure 14. ALE ARS of ISO 19901 code, average GM, and ETAF for SPD2.

time was obtained as short amount of time, which according to Mashayekhi and Estekanchi [32], target time cannot satisfy effective number of cycles in ET analysis. Thus, the ETAF was scaled by coefficient of 0.25 ; target times in which the ET spectrum had compatible area with ELE and ALE are displayed in Table 3 .

The average of seven scaled records, the average of three ETAF, and $S_{a}$ related to ELE level for SP are shown in Figure 11, and those for SPD2 are shown in Figure 13. Aforementioned graphs related to the ALE level of SP are illustrated in Figure 12, and those for SPD2 are shown in Figure 14.

As shown in Figures 11 to 14, ISO scaling method provides a reasonable match in range of $0.2 T_{n}$ to $1.5 T_{n}$ for these long period structures; moreover, the average of ETAFs has good match with the average of real GMs. According to this match, it is expected that the ET method can estimate the responses of these structures in different levels.

Table 3. Equivalent time of hazard level of ELE and ALE for ET records.

\begin{tabular}{cccccc}
\hline & & \multicolumn{2}{c}{ Equivalent time for different hazard } & \multirow{2}{*}{$\begin{array}{c}\text { Average } \\
\text { levels (s) }\end{array}$} \\
\cline { 3 - 5 } & & \multicolumn{3}{c}{ ETAme } \\
\cline { 3 - 5 } SP & ALE & 17.70 & 17.70 & 17.00 & 17.47 \\
& ELE & 12.60 & 12.40 & 13.80 & 12.93 \\
\multirow{2}{*}{ SPD2 } & ALE & 12.5 & 13 & 14 & 13.17 \\
& ELE & 8.9 & 9.11 & 8.23 & 8.75 \\
\hline
\end{tabular}




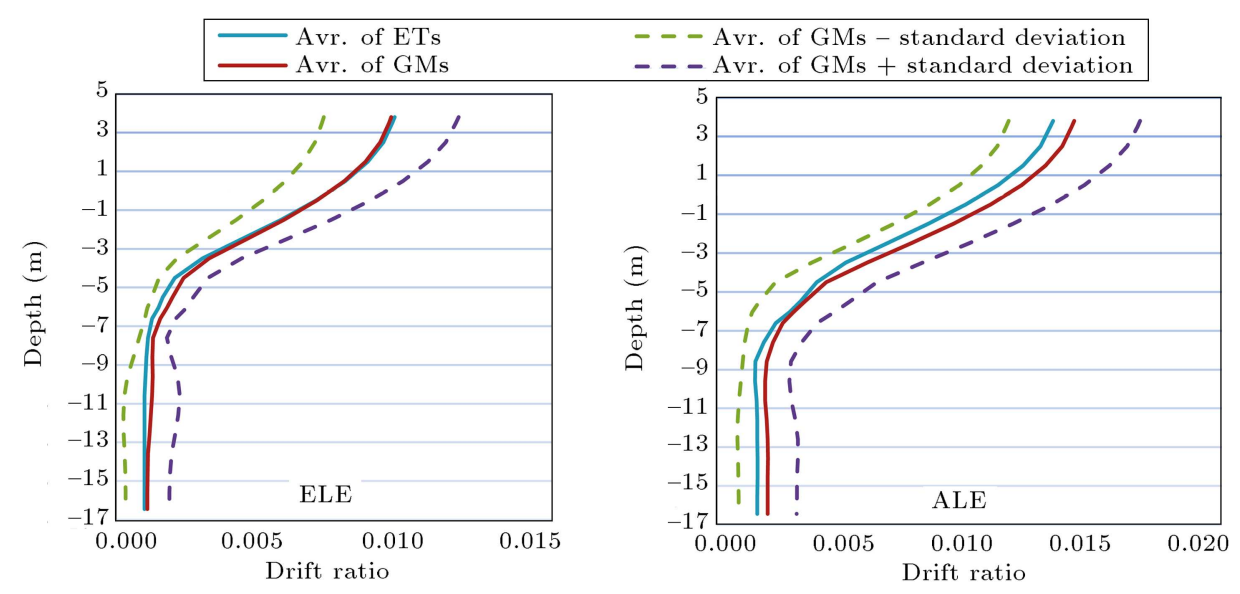

Figure 15. Average inter-story drift ratios of SP under GMs and ET accelerograms.

\section{Results and discussion}

For investigating the accuracy and proximity of the estimation in the ET method with the selected records, we compared the results of average ET analysis obtained from the ETA20en01-3 with those of structures subjected to the GMs mentioned in Table 1. The behaviors of the SP and SPD2 were evaluated under GMs and ET acceloregrams, and responses are shown in Figures 15 and 16.

As shown in Figures 15 and 16, the ET estimations of the response of structures with deep foundations are satisfactory. These figures indicate appropriate estimation of ET method with mean difference of $10 \%$ and the max difference of $20 \%$ compared to average results of GMs. It is noteworthy that the results of ET method are well within the range of average results of real earthquake records, considering a standard deviation. Moreover, this lack of exact match is partially caused due to the random nature of acceleration functions, and this is also true in the case of GMs.

The results of ET analysis are presented by

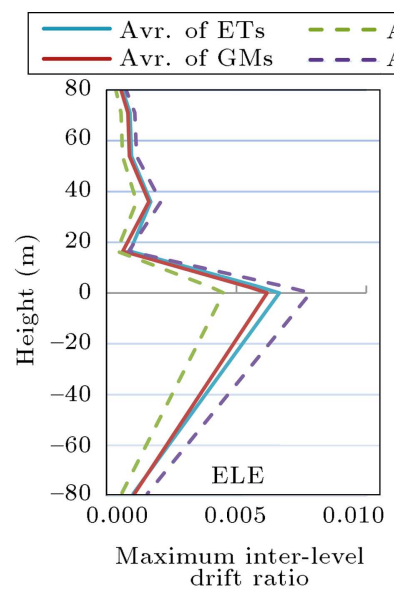

r. of GMs - standard deviation

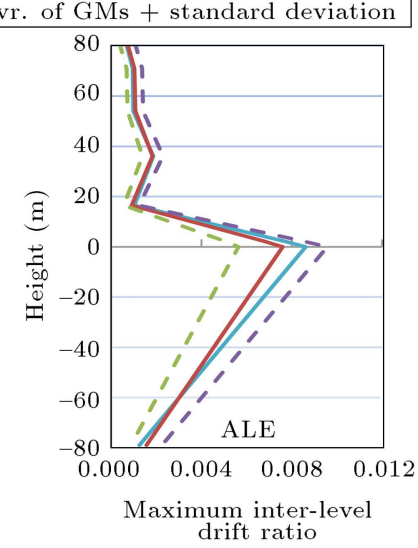

Figure 16. Average inter-story drift ratio of SPD2 under GMs and ET accelerograms. increasing ET curves where they are coordinated at each time value, $t$, corresponding to the maximum absolute value of the considered variables from $t=$ 0 to the desired time. As a result of statistical characteristics of the ET analysis, the ET curves are usually serrated. To solve this problem, we used a moving average procedure to reduce the serrated nature of ET curves. To obtain the final ET curves, the ET curves obtained from three acceleration functions were first averaged; afterwards, the procedure of moving average was applied to serrated ET curves. Figure 17 shows the performance curve from the ET method for $\mathrm{SP}$, where EDP is the drift ratio; moreover, Figures 18 to 21 illustrate the performance curve by the ET method for SPD2.

As mentioned earlier, one of the advantages of ET method is that it can show simplified results. Hence, we chose more than one EDP for the JTOP. Because the structure of platform is a pile-supported system, we used some EDPs considered for these features. The first EDP was the maximum inter-level drift ratio by considering the pile, and the performance curve is shown in Figure 18. The second EDP was the maximum pile drift ratio; this EDP occurred with a particular mechanism, where the height of drift is

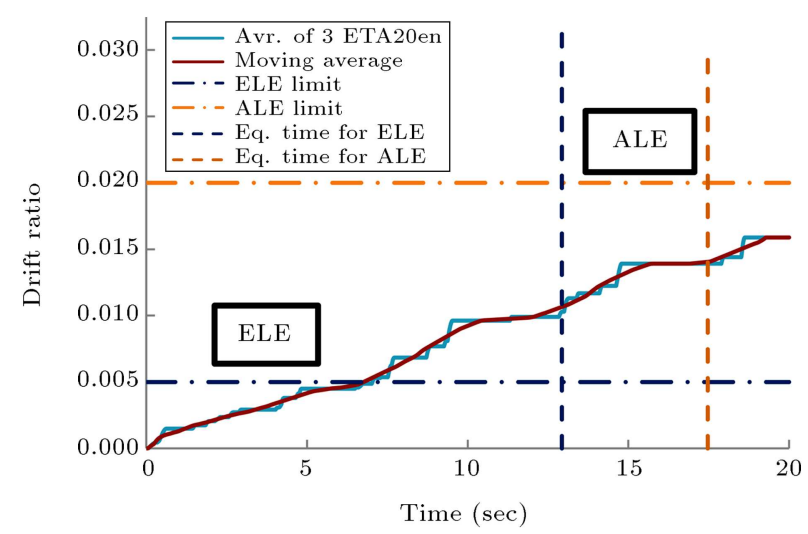

Figure 17. SP performance curve for EDP of drift ratio. 


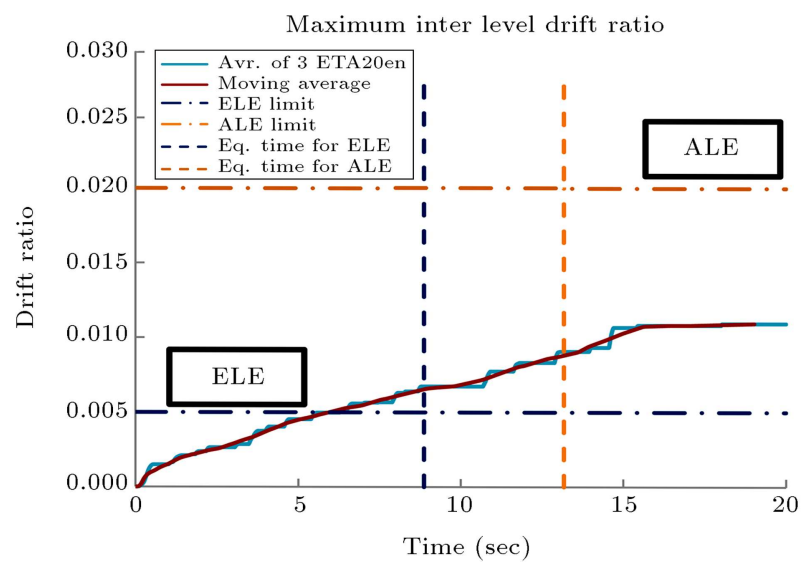

Figure 18. SPD2 performance curve for EDP of maximum inter-level drift ratio.

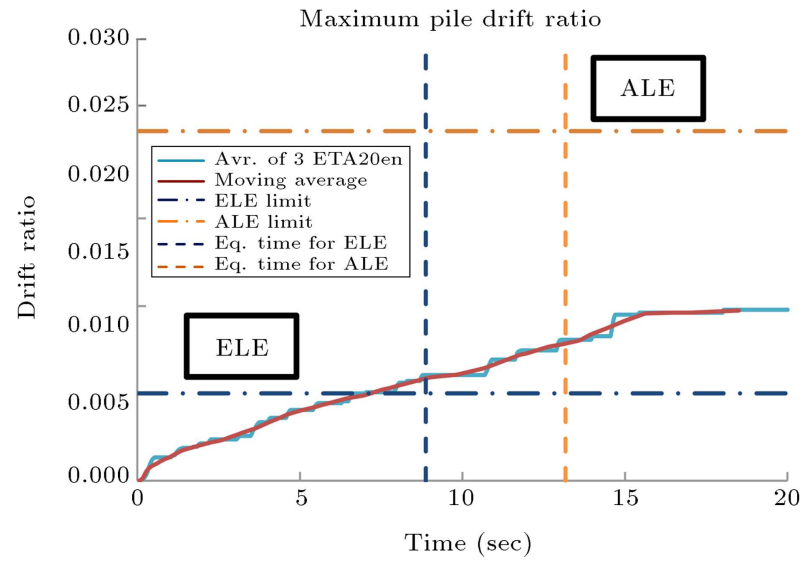

Figure 19. SPD2 performance curve for EDP of maximum pile drift ratio.

between two hinges: The first hinge occurs at the depth where the first plastic hinge forms due to the maximum moment. The second hinge occurs just below the first level or at the mud line level. The performance curve of this EDP is shown in Figure 19. The third EDP was the maximum drift ratio which is the max of two previously EDPs, and the performance curve is shown in Figure 20. The fourth EDP which was only for pile is the maximum pile displacement performance curve, as shown in Figure 21 [33].

As is obvious from Figures 18 to 21, according to what is alluded to for the benefits of ET method, ET method not only shows results in a simpler mode, but also facilitates comparison between the EDPs. In this case, the EDP of maximum pile displacement was dominant EDP within the EDPs because in this EDP, the increasing ET curve crossed ELE limit sooner than other EDPs.

Moreover, it can be concluded that this structure cannot satisfy ELE event since not only maximum pile displacement, but also other EDPs crossed ELE limit before equivalent time for ELE in the graphs. However, SPD2 fulfilled ALE event because none of

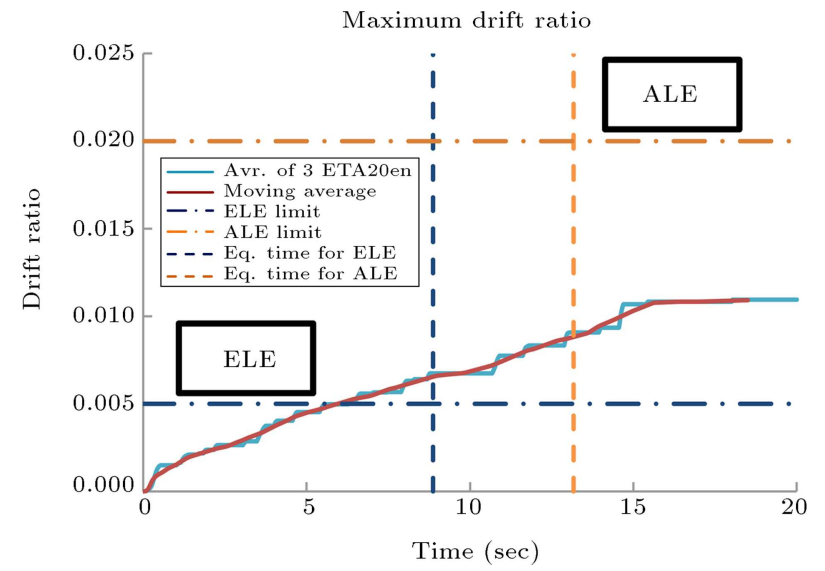

Figure 20. SPD2 performance curve for EDP of maximum drift ratio.

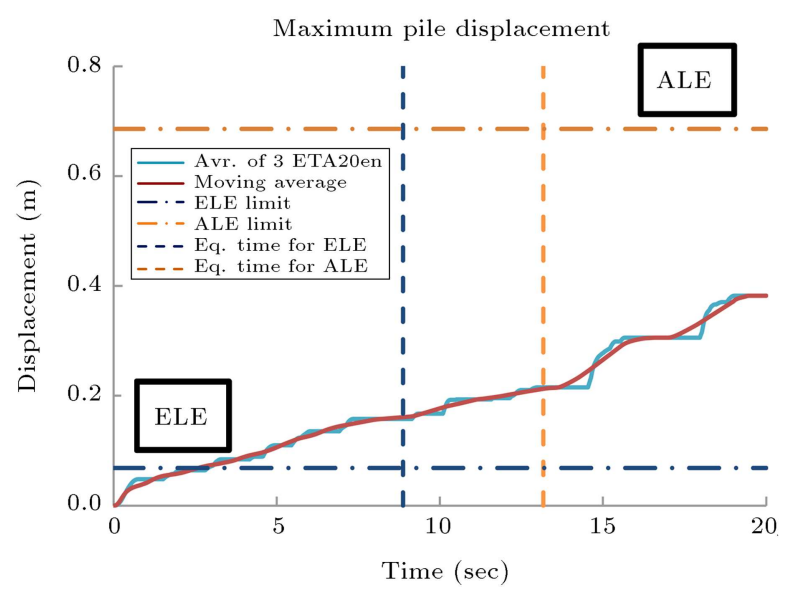

Figure 21. SPD2 performance curve for EDP of maximum pile displacement.

the increasing ET curves crossed ALE limit before equivalent time for ALE limit.

\section{Conclusion}

In this paper, we investigated application of ET method through nonlinear seismic analysis of offshore pile supported systems, and responses of these structures under grounds motions and ETAF were compared. The following conclusions can be drawn:

1. The drift ratios of ET analyses for both the single pile and the jacket-type offshore platform were compared well with those from GMs in these structures, and the results indicate a proper approximation of ET method compared to conventional time-history method;

2. Artificial acceleration functions of ET method performed reliably in cases involving deep soils and pile supported structures in this study. ETAFs produced acceptable results compared to real ground motions while passing different soil layers; 
3. Different EDPs for the JTOP were considered, and it is shown that ET method provides simplified presentation of multi-level seismic evaluation so that results can be compared in different EDPs conveniently;

4. In this study, due to the presence of piles, each record had to be applied at the bottom of the pile system, and pass through different layers of soil. This requirement heavily increases the computational demand required for conventional nonlinear time-history analysis. By applying ET method, this required computational demand can be considerably reduced.

\section{Nomenclature}

$\alpha \quad$ An optimization weighting parameter

$\sigma_{m}^{\prime} \quad$ Mean effective stress

$\sigma_{v c}^{\prime} \quad$ Vertical effective stress

$a_{g}$

ALE Abnormal Level Earthquake

ARS Acceleration Response Spectra

BNWF Beam on Nonlinear Winkler

Foundation

$C_{r} \quad$ Seismic reserve capacity factor

$C_{u} \quad$ Coefficient of uniformity

$c_{u} \quad$ Undrained shear strength

$D_{50} \quad$ Median particle size diameter

$D_{r} \quad$ Relative density

EDP Engineering Demand Parameter

EI $\quad$ Flexural stiffness

ELE Extreme Level Earthquake

Eq Equivalent

ET Endurance Time

ETAF Endurance Time Acceleration Function

$F\left(a_{g}\right) \quad$ Optimization target function

$G \quad$ Shear modulus

$G_{\max } \quad$ Maximum shear modulus

GM Ground Motion

JTOP Jacket Type Offshore Platform

$K_{0} \quad$ Coefficient of lateral stress at rest

$K_{2, \max } \quad$ parameter relating $G_{\max }$ and $\sigma_{m}^{\prime}$ and a function of density or void ratio

$L \quad$ Length of member

LL Liquid Limit

MRDF Modulus reduction curve and damping curve including reduction factor

$P_{\text {atm }} \quad$ Atmospheric pressure

PEER Pacific earthquake research center
PI Plasticity Index

$S_{a} \quad$ Spectral acceleration

$S_{a}(T, t) \quad$ Acceleration response for period $T$ at time $t$

$S_{a c}(T) \quad$ Code acceleration response for period $T$

$S_{a T}(T, t)$ Target acceleration response at time $t$

$S_{u}(T, t) \quad$ Displacement response for period $T$ at time $t$

$S_{u T}(T, t) \quad$ Target displacement response at time $t$

SP Single Pile

$T \quad$ Period of free vibration

$t_{\text {Target } \quad \text { Target time }}$

$T_{\max } \quad$ Maximum free vibration period (sec) to be considered in the optimization

$t_{\max } \quad$ Time corresponding to the end of acceleration function

$T_{n} \quad$ Fundamental period of structure

\section{References}

1. Seif, M.S., Mehdigholi, H., Estekanchi, H.E. and Dastan, M.A. "Evaluation of seismic behavior of fixed offshore platforms with endurance time method", International Journal of Maritime Technology, 15, pp. 97-103 (2012).

2. Estekanchi, H.E., Vafai, A. and Sadeghazar, M. "Endurance time method for seismic analysis and design of structures", Scientia Iranica, 11, pp. 361-370. (2004).

3. Estekanchi, H.E., Riahi, H.T. and Vafai, A. "Application of endurance time method in seismic assessment of steel frames", Engineering Structures, 33, pp. 25352546 (2011).

4. Riahi, H.T., Estekanchi, H.E. and Boroujeni, S.S. "Application of endurance time method in nonlinear seismic analysis of steel frames", Procedia Engineering, 14, pp. 3237-3244 (2011).

5. Valamanesh, V., Estekanchi, H.E., Vafai, A. and Ghaemian, M. "Application of the endurance time method in seismic analysis of concrete gravity dams", Scientia Iranica, 18, pp. 326-337 (2011).

6. Alembagheri, M. and Estekanchi, H.E. "Seismic assessment of unanchored steel storage tanks by endurance time method", Earthquake Engineering and Engineering Vibration, 10, pp. 591-603 (2011).

7. Estekanchi, H.E. and Alembagheri, M. "Seismic analysis of steel liquid storage tanks by endurance time method", Thin-Walled Structures, 50, pp. 14-23 (2012).

8. Tavazo, H., Estekanchi, H.E. and Kaldi, P. "Endurance time method in the linear seismic analysis of shell structures", International Journal of Civil Engineering, 10, pp. 169-178 (2012). 
9. Boulanger, R., Curras, C., Kutter, B., Wilson, D. and Abghari, A. "Seismic soil-pile structure interaction experiments and analyses", Journal of Geotechnical and Geoenvironmental Engineering, 125, pp. 750-759 (1999).

10. Mazzoni, S., McKenna, F., Scott, M.H. and Fenves, G.L. OpenSees Command Language Manual (2007).

11. Hashash, Y.M.A., DEEPSOIL V 5.0, User Manual and Tutorial, 2002-2011, University of Illinois at Urbana-Champaign (2011).

12. ISO. "Petroleum and natural gas industries-specific requirements for offshore structures (ISO 19901-2)", Part 2, Seismic Design Procedures and Criteria, Switzerland, International Standard Organization (2004).

13. Wilson, D.W., Boulanger, R.W. and Kutter, B.L. "Soil-pile-superstructure interaction at soft or liquefiable soil sites - centrifuge data report for CSP4, UCD/CGMDR-97/05", Center for Geotechnical Modeling, Department of Civil and Environmental Engineering, University of California, Davis, CA (1997).

14. Wilson, D.W., Soil-Pile-Superstructure Interaction in Liquefying Sand and Soft Clay, UCD/CGM-98/04, University of California, Davis (1998).

15. Wang, S., Kutter, B.L., Chacko, M.J., Wilson, D.W., Boulanger, R.W. and Abghari, A. "Nonlinear seismic soil-pile structure interaction", Earthquake Spectra, 14, pp. 377-396 (1998).

16. Matlock, H., Bryant, L.M. and Foo, S.H.C. "Simulation of lateral pile behavior under earthquake motion", Earthquake Engineering and Soil Dynamics, Pasadena, CA, American Society of Civil Engineers, pp. 600-19 (1978).

17. Penzien, J., Scheffey, C.F. and Parmelee, R.A. "Seismic analysis of bridges on long pile", Journal of the Engineering Mechanics Division, 90, pp. 223-254 (1964).

18. Kagawa, T. and Kraft, L.M. "Seismic $\mathrm{p} \sim \mathrm{y}$ responses of flexible piles", Journal of the Geotechnical Engineering Division, 106, pp. 899-918 (1980).

19. Nogami, T., Otani, J., Konagai, K. and Chen, H. "Nonlinear soil-pile interaction model for dynamic lateral motion", Journal of Geotechnical Engineering, 118, pp. 89-106 (1992).

20. Naggar, M.H.E. and Bentley, K.J. "Dynamic analysis for laterally loaded piles and dynamic p-y curves", Canadian Geotechnical Journal, 37, pp. 1166-1183 (2000).

21. API., Recommended Practice for Planning, Designing and Constructing Fixed Offshore Platforms-Working Stress Design (API RP2A-WSD), Washington (DC), American Petroleum Institute (2005).

22. Seed, H.B. and Idriss, I.M., Soil Moduli and Damping Factors for Dynamic Response Analyses, In Earthquake Engineering Research Center, EERC 70-10: College of Engineering, University of California (1970).
23. Phillips, C. and Hashash, Y.M.A. "Damping formulation for nonlinear 1D site response analyses", Soil Dynamics and Earthquake Engineering, 29, pp. 114358 (2009).

24. Darendeli, M.B. Development of a New Family of Normalized Modulus Reduction and Material Damping Curves, University of Texas at Austin (2001).

25. Vucetic, M. and Dobry, R. "Effect of soil plasticity on cyclic response", Journal of Geotechnical Engineering, 117, pp. 89-107 (1991).

26. Stewart, J.P., Benchmarking of Nonlinear Geotechnical Ground Response Analysis Procedures, Pacific Earthquake Engineering Research Center (2008).

27. Van Laak, P.A., Taboada, V.M., Dobry, R. and Elgamal, A.-W. "Earthquake centrifuge modeling using a laminar box", In Dynamic Geotechnical Testing II. ASTM Special Technical Publication: ASTM STP 1213, p. 15 (1994).

28. Tabandeh, S.A., Seismic Assessment of Fixed Jacket Type Offshore Platforms - Special Attention to Record Selection and Probabilistic Nonlinear Site Response Analysis, Sharif University of Technology (2010).

29. http://cgm.engr.ucdavis.edu/, Center for Geotechnical Modeling at University of California Davis.

30. ISO, Petroleum and Natural Gas Industries - Fixed Steel Offshore Structures (ISO 19902), Switzerland, International Standard Organization (2007).

31. Pacific Earthquake Engineering Research Center, PEER Ground Motion Database, Beta Version. http://cgm.engr.ucdavis.edu/

32. Mashayekhi, M. and Estekanchi, H.E. "Significance of effective number of cycles in endurance time analysis", Asian Journal of Civil Engineering (Building and Housing), 13, pp. 647-57 (2012).

33. Ebrahimian, H., Assessment of Existing Jacket-Type Offshore Platforms in the Persian Gulf Region for Service Life Extension on Operative Conditions, Sharif University of Technology (2012).

\section{Biographies}

Hamed Hasani received his MS degree from Sharif University of Technology, Tehran, Iran. His research interests include earthquake engineering, offshore structures, and soil-pile-superstructure interaction.

Ali Akbar Golafshani graduated in 1978 from Utah State University, Logan, USA with a BS degree. He received his $\mathrm{MS}$ and $\mathrm{PhD}$ degrees in the field of Structural Mechanics from the University of California, Berkeley, USA in 1979 and 1981. Between 1982 and 2016, he had cooperation with Sharif University of Technology as a Professor in the Department of Civil Engineering. He passed away in April 2016.

Homayoon Estekanchi is a Professor of Civil En- 
gineering at Sharif University of Technology (SUT), Tehran, Iran. He received his $\mathrm{PhD}$ degree in Civil Engineering from SUT in 1997 and has been a faculty member at SUT since then. He is a member of Iranian Construction Engineers Organization, ASCE, Iranian
Inventors Association, and several other professional associations. His research interests include a broad area of topics in structural and earthquake engineering with a special focus on the design of tall buildings and industrial structures. 